\title{
"Resiliency Engagement and Care in Health (REaCH); a Telephone Befriending Intervention for Up Skilled Rural Youth in the Context of COVID- 19 Pandemic: Study Protocol for a Multi-centre Cluster Randomized Controlled Trial".
}

\section{D Saju ( $\nabla$ saju@rajagiri.edu )}

Rajagiri College of Social Sciences https://orcid.org/0000-0002-6188-9267

Komal Preet ALLAGH

Rajagiri College of Social Sciences

Anuja Maria Benny

Rajagiri College of Social Sciences

Lorane Scaria

Rajagiri College of Social Sciences

Natania Cheguvera

Rajagiri College of Social Sciences

Sunirose IP

Rajagiri College of Social Sciences

Study protocol

Keywords: Randomised control trial, COVID-19, befriending, India

Posted Date: March 25th, 2021

DOl: https://doi.org/10.21203/rs.3.rs-55429/v1

License: (c) (i) This work is licensed under a Creative Commons Attribution 4.0 International License. Read Full License

Version of Record: A version of this preprint was published at Trials on July 28th, 2021. See the published version at https://doi.org/10.1186/s13063-021-05465-5. 


\section{Abstract}

\section{Background}

The lockdown associated with the COVID-19 pandemic is likely to impact the mental health of people, especially those who are from economically backward and vulnerable sections of society. Mental health can be affected from various reasons including fear of disease transmission, from response measures like social distancing and lockdown, fear of being in quarantine, loneliness, depression due to isolation, fear of losing work and livelihood and avoiding health care due to fear of being infected. Telephonic befriending intervention by non-specialists will be used to provide social and emotional support to the youth from the Deen Dayal Upadhyaya Grameen Kaushalya Yojana (DDUGKY), an initiative of Government of India. This study aims to promote mental wellbeing and reduce depressive symptoms by assisting participants to mobilize social support from family, friends and significant others by using the telephonic befriending intervention.

\section{Methods}

In this article, we report the design and protocol of a multi-centre cluster randomised controlled trial. In total, 1440 participants aged 18-35 years, who have recently passed out of the DDU-GKY initiative will be recruited in the study, from 12 project implementing agencies across 6 geographical zones of India. Participants from 6 of these agencies will be assigned into the telephonic befriending intervention arm and the other 6 agency participants will be assigned to the general enquiry phone call arm (control). The primary outcomes of this study are mental wellbeing, depressive symptoms and perceived social support. Baseline assessments and follow-up assessments will be carried out 1 month following the intervention using WHO-5, PHQ and MSPSS-12 questionnaires. The befriending intervention will be provided by DDUGKY staff who will be trained by a virtual training program.

\section{Discussion}

The results of this trial will help assess whether participants who are offered emotional, social and practical support through befriending, will experience lesser symptoms of depression and better mental health compared to participants who do not receive this intervention. And whether participants who receive the intervention will perceive better social support.

\section{Trial registration}

This intervention trial This intervention trial is registered under Clinical Trial Registry India (ICMR-NIMS) on $27^{\text {th }}$ July 2020; registration number: CTRICTRI/2020/07/026834

\section{Background}

In India, the first case of COVID-19 was confirmed from Kerala on 30th January 2020 [1]. On the 11th of March 2020, coronavirus disease (COVID-19) was declared a pandemic by the World Health Organization 
(WHO) [2]. Kerala declared a state-wide lockdown on the 23rd March 2020, to prevent the community transmission of this virus [3]. As a result of the lockdown, people were enforced to stay in their homes, which is very likely to impact their mental and physical health. The unpredictability and uncertainty of controlling the disease, along with misinformation around it, will increase mental stress and concern among the people [4]. Recent studies have shown that even with a short term lockdown, people report symptoms of mental stress and mental disorders like anxiety, depression, insomnia and post-traumatic stress disorder $[5,6]$. Findings from a recent online survey conducted among residents of New Delhi revealed $55.3 \%$ of participants have sleep disturbances during the lockdown, $63.4 \%$ report work and income being severely affected, $7 \%$ report anxiety and restlessness and $12.1 \%$ felt helpless and depressed [7]. Thus, the pandemic is not just a threat to a country's physical health but will also affect the mental, social and economic well-being.

A survey by the Indian society of Labour economics showed job loss as the most severe immediate impact of COVID-19, while lower economic growth and rise in inequality as the probable long-term effects [8]. Loss of employment and the falling economy is expected to have a huge impact on mental health, especially those individuals living under precarious conditions. During such time, people experience fear, anxiety, stress and other challenges that can trigger common mental health disorders [9].

The Ministry of rural development (MoRD), Government of India (GOI), launched an initiative in 2014 called the Deen Dayal Upadhyaya Grameen Kaushalya Yojana (DDU-GKY), to add diversity to the incomes of the rural poor families and to cater to the career aspirations of rural youth [10]. This initiative is present in 28 States of India and focuses on youth from poor rural families (aged 15 to 35 years). Currently, 1,575 projects are being implemented by over 717 partners across India [10].

The Rajagiri College of Social Sciences, in Cochin, Kerala, is one of the projects implementing agencies (PIA), with 1500 students passed out from 2016 till date, under the DDU-GKY initiative. In this paper, we present the study protocol of the REaCH (Resiliency Engagement and Care in Social Health) intervention trial. The ReaCH intervention trial involves using friendly telephonic interactions as an intervention for extending psychosocial support and creating a platform for sharing concerns of the youth, passed out from the DDU-GKY initiative, especially in the context of COVID-19 pandemic and the associated lockdown.

People with socioeconomic vulnerability are more prone to common mental disorders $[11,12,13]$, mainly due to isolation from inadequate social support and the absence of holistic health services [14]. Individuals from economically backward and vulnerable sections of the society have shown to appreciate and benefit from emotional and social support $[15,16]$. One way of providing this support is through befriending, which has proven to be effective in improving mental health and reducing depression [15]. Befriending is defined as an intervention aimed at providing the client with additional social support through the development of an empathic, non-judgmental, non-directive, affirming and purposeful relationship [15]. 
In an epidemic outbreak, psychosocial interventions are essential [17] and social support has shown to have a direct relationship with positive mental health and acts as a buffer for various stressors affecting mental health [18]. People with strong social relationships enjoy a sense of security and belongingness to a network of systems that acts as a protective factor against various mental health issues [19].

Befriending is most often delivered by lay personnel or non-specialist workers and [20] found that their use in the care of subjects with common mental disorders was not only cost-effective but also costsaving. In our trial, the intervention will be provided by DDU-GKY staff, which will enhance the use of existing staff, reduce cost, improve sustainability as the staff will be empowered to identify similar mental health issues in students in the future and increase acceptability as the staff are always available. Before the main trial, we plan a pilot trial among the students of the PIA at Rajagiri University, Kerala. Following the pilot trial, Rajagiri University team will lead the trial across 28 States of India, with the support of the Ministry of Rural Development. This nationwide telephonic befriending intervention is set out to address immediate priorities and strategies for providing emotional and practical support to the vulnerable people, in the context of COVID-19 related lockdown. We expect that participants whom we offer emotional, social and practical support through befriending, will experience lesser symptoms of depression and better mental health compared to participants who do not receive this intervention. And participants who will receive the intervention will report better social support than the control group.

\section{Methods}

\section{Aim}

The aim of the befriending intervention is to promote mental wellbeing and reduce depressive symptoms by assisting participants to mobilize social support from family, friends and significant others.

The objectives of this trial are:

- To proactively engage with students to hear their concerns in a non-judgmental manner and empathize with them.

- To reassure students and get them back to work/ find a job and normal patterns of life, as early as possible

- To create a database of students who have either lost their jobs or are in search of jobs and link them to the job market.

- To facilitate active linkage with various formal and informal resources to reduce their distress

\section{Design}

Cluster Randomised controlled trial

\section{Outcomes}


1. Mental wellbeing measured by World Health Organization-Five Well-being Index (WHO-5) [21, 22]

2. Depressive symptoms measured by the Patient Health Questionnaire (PHQ-9) [23].

3. Perceived social support measured by the Multidimensional Scale of Perceived Social Support (MSPSS-12) [24]

\section{Study location}

For feasibility reasons, recruitment will be restricted to 6 geographical zones of India. These divisions are selected in consultation with Director of National Institute for Rural Development and Panchayat Raj (NIRDPR), training head, and her team. This clustering was done to capture the sociocultural and economic variations in settings and population and to address the needs specific to each zone. Table 1 lists the states that fall under each zone.

Table 1

List of states that fall under each zone.

Geographical States in the zone
zones

1. Northern zone Delhi, Himachal Pradesh, Jammu \& Kashmir, Punjab, Uttarakhand, Uttar Pradesh

2. North-East Assam, Arunachal Pradesh, Manipur, Meghalaya, Mizoram, Nagaland, Sikkim, zone Tripura

3. Central zone Madhya Pradesh, Chattisgarh

4. Eastern zone Bihar, Jharkhand, Odisha, West Bengal

5. Western zone Goa, Gujarat, Maharashtra

6. Southern Andhra Pradesh, Karnataka, Kerala, Puducherry, Tamil Nadu, Telangana zone

\section{Recruitment of clusters (PIAs)}

Two project implementing agencies (PIA) will be recruited from each of the six zones (12 PIAs in total). For a PIA to be included in this study, it should be in one of the six zones and should be an active, currently operational PIA with a full-time staff. A computer-generated randomisation procedure will be used by the NIRDPR to select the PIAs in the six zones. The NIRDPR will contact only those agencies meeting the inclusion criteria, and invite them to be a part of the study. Six of the selected PIAs will be allocated to the intervention arm and 6 PIAs will be allocated to the control arm. Information on the number of full-time staff, contact details of passed out students will be collected by the State monitoring agencies.

\section{Duration}


The total duration of the trial is four weeks. The trial will run parallelly across all six zones. The expected duration of involvement of each participant is 1 month.

\section{Recruitment of participants within PIAs}

We will recruit 120 passed out youth from each participating PIA (total=1440). All participants from one PIA will be either in the intervention arm or control arm. The participants are aged between 18-35, all genders, and up skilled in different domains. Participants will be invited to take part in baseline assessment and intervention programs.

\section{Inclusion criteria}

1. Alumni students of the DDU-GKY program, who are either working or are in search of a job

2. Own a smartphone

\section{Exclusion criteria}

1. Do not possess a smartphone

2. Unable to operate a smartphone

\section{Randomisation and blinding}

Following the completion of the baseline assessment for all participants, the eligible participants will be randomised at 1:1 ratio to one of the two arms (intervention or control) by the trial manager based on the PIA to which they belong. Computer-generated random number list will be created by a computer technician and odd numbers on the list will be allocated to the intervention arm and the even numbers will be allocated to the control arm.

\section{Design measures to avoid bias}

The allocation codes will be concealed by the use of a computer-generated random number list. The principal investigator, the trial team, trial manager or staff members will be blind to the allocation codes during the trial. The control arm will also receive general enquiry phone calls that will further help in masking the allocation. To avoid contamination, we will physically separate the intervention team and the control team, and blind the telephone support person (staff) about which arm they belong to. We believe the lockdown and work from home will assist in masking. Separate staff will make the phone calls in the intervention and control arms.

\section{Assessments and procedures}

Baseline assessments will be administered to all participants through an online survey by the research team. Questionnaires include PHQ-9, MSPSS, WHO-5 and socio-demographics. These questionnaires will be translated into the local languages and back-translated for accuracy. Detailed instructions and sufficient explanations will be provided on the initial page of the online survey. 
The PHQ-9 questionnaire will be used to measure depression, with scores of 1-4, 5-9, 10-14, 15-19, $20-27$ indicating minimal, mild, moderate, moderately severe and severe depressive symptoms. WHO- 5 questionnaire will be used to measure wellbeing and the total row score (ranging from 0 to 25) is multiplied by 4 to provide the final score. 0 represents the worst possible wellbeing and 100 represents the best possible wellbeing. The MSPSS will be used to measure perceived social support from three sources: family, friends, and a significant other. This measure contains 12 questions which are rated on a 7-point scale as 'very strongly disagree', 'strongly disagree', 'mildly disagree', 'neutral', 'mildly agree', 'strongly agree', and 'very strongly agree'.

Post-intervention follow-up assessment will be carried out through telephone for both control and intervention arm. This will use the same baseline survey assessment tools and will be performed online.

\section{Training of the staff}

The chief Executive officer and the chief operating officer/ State program manager from the six selected PIAs (12 in total) will be trained by the expert resource persons on content, process and strategies of the intervention manual. Training of trainers will be given to 36 facilitators, 6 members each from 6 intervention PIAs and the State Rural Livelihood Mission (SRLM) from the selected States. A virtual master class will be held to train these facilitators on providing the intervention (telephonic befriending). This training will last for approximately six hours and will include a session on question and answers. Following this training, the training video, protocol booklet, and frequently asked Q\&As will be shared with the facilitators. Additionally, an online support centre will be set-up for continuous support to the trainers.

The trained facilitators will train 6 PIA staff (two from each agency) and each trained PIA staff will train an average of 6 other staff members. Staff who are a part of the DDU-GKY project for one year or longer will be trained to deliver the intervention. Staff who are a part of the intervention team will only receive this training on the intervention module. These trained staff members will reach out to 720 DDU-GKY alumni through this intervention.

Each befriender will be allocated between 10 to 15 students in their PIA. A 6-hour virtual training is designed for the staff, covering the components to be delivered during each session. An intervention manual for the training has been developed, which was also translated into the local language. After the training has been completed, the trained staff will be provided with the intervention manual, online video of the training session with subtitles, audio clips of sample interview content and a module on frequently asked questions. The intervention manual includes guidelines on developing a relationship with the client, introduction and orientation of participant to befriending, management of participant distress, confidentiality and safety issues for both staff and participants.

Separate staff members will be involved in the intervention arm and control arm. No specific training will be given for the staff making the telephonic calls in the control arm. They will provide general information about the precautions that need to be taken to protect themselves from the infection, and inquire about how the family is coping with the lockdown related issues. 


\section{Intervention}

The two arms of the trial are:

1. Telephone befriending intervention arm $(n=720)$

2. General enquiry phone call arm (control) $(n=720)$

\section{Intervention arm: Telephone befriending intervention}

This involves participants receiving befriending from trained DDU-GKY staff, through one-to-one phone calls which they will receive in their homes, at a time convenient to them. The telephonic befriending intervention will be conducted in three phases: (i) Proactive engagement and crisis intervention, (ii) Problem-solving oriented support therapy and (iii) Assertive linkage with community resources (see table 1). The three phases will spread across four phone calls for 30 minutes to 1-hour duration. Participants will be contacted by their telephone support person, and an introductory session will be arranged over the phone at each participants' convenient time to establish a rapport. 
Table 2

Three phases of the structured befriending intervention

\begin{tabular}{|c|c|c|}
\hline Phase & Objectives & Interventions \\
\hline \multirow{5}{*}{$\begin{array}{l}\text { Phase 1: Proactive } \\
\text { engagement and crisis } \\
\text { intervention }\end{array}$} & \multirow{5}{*}{$\begin{array}{l}\text { - To provide } \\
\text { emotional support } \\
\text { - To provide } \\
\text { strategies to alleviate } \\
\text { distress and crisis }\end{array}$} & In this session the befriender will: \\
\hline & & $\begin{array}{l}\text { - Establish a positive and supportive } \\
\text { relationship with the participant }\end{array}$ \\
\hline & & - Provide emotional support \\
\hline & & $\begin{array}{l}\text { - Psycho-education about COVID-19 including } \\
\text { precautionary measures, }\end{array}$ \\
\hline & & $\begin{array}{l}\text { - Enquire about general health and biological } \\
\text { functioning. }\end{array}$ \\
\hline \multirow{6}{*}{$\begin{array}{l}\text { Phase 2: Problem- } \\
\text { solving oriented } \\
\text { supportive therapy }\end{array}$} & \multirow{6}{*}{$\begin{array}{l}\text { - To provide } \\
\text { strategies to solve } \\
\text { client's problems }\end{array}$} & This includes five general stages: \\
\hline & & $\begin{array}{l}\text { - Problem orientation: Helping participant to } \\
\text { identify the most distressing problem }\end{array}$ \\
\hline & & $\begin{array}{l}\text { - Problem definition and formulation: defining } \\
\text { the problem based on its impact on day to day } \\
\text { functioning }\end{array}$ \\
\hline & & $\begin{array}{l}\text { - Generation of alternatives: Helping the } \\
\text { participant to identify alternative options }\end{array}$ \\
\hline & & - Assist the decision-making \\
\hline & & - Propose strategies to implement \\
\hline \multirow{4}{*}{$\begin{array}{l}\text { Phase 3: Assertive } \\
\text { linkage with } \\
\text { community resources }\end{array}$} & \multirow{2}{*}{$\begin{array}{l}\text { - To utilize } \\
\text { community resources } \\
\text { for the well-being }\end{array}$} & In this session, the befriender will: \\
\hline & & - Provide practical support and information; \\
\hline & \multirow{2}{*}{$\begin{array}{l}\text { - To solve a crisis like } \\
\text { job loss, financial } \\
\text { insecurity, etc. }\end{array}$} & $\begin{array}{l}\text { - Emphasize on social support and will } \\
\text { provide strategies to improve it, }\end{array}$ \\
\hline & & $\begin{array}{l}\text { - Reassurance to the participant before } \\
\text { termination of the session. }\end{array}$ \\
\hline
\end{tabular}

During the telephone calls, a semi-structured questionnaire will be used to gather information on the stressors and risk factors in the context of this pandemic outbreak. Telephonic conversations will be recorded with the consent of the participants and later transcribed by the research team. The specific domains that will be assessed include the situational stressors in the context of COVID-19 outbreak and the different support mechanisms available to manage the difficulties. The staff will be trained to encourage the participant to speak about their issues and difficulties in the present context and empathetically listen to their worries. 


\section{Control arm: General enquiry phone calls}

Participants randomised to the control arm will not be receiving any intervention. However, they will participate in a baseline and follow up assessment using the same instruments. They will receive 4 general enquiry phone calls lasting 5 to 30 minutes. It will be a general inquiry about the precautions that need to be taken to protect themselves from the infection, and how the family is coping with the lockdown related issues. The main focus will be given on psycho-education based inquiries on COVID-19.

\section{Data entry and data management}

The online data collection tool was developed and was linked to the dedicated server kept in the research centre. The participant will enter the data directly to the online mobile portal which will upload the data directly onto the research server. The stored data set and codes will be protected using a password by the trial manager, who is independent of the trial team. The centrally stored data will be cleaned and analysed by the research team under the supervision of the trial manager and the trial manager will verify the results independently to ensure the accuracy of the results. Data will be re-identified to ensure the confidentiality of information. Major findings of the study will be reported in scientific journals and will be presented in conferences and workshops. Interested researchers can contact the Principal Investigator for further research collaborations.

\section{Sample size and statistical analysis}

A target sample size of 1440 participants (720 in each arm) with less than $5 \%$ loss to follow up after one moth of intervention estimated to have $80 \%$ power. All participants will be included in the analysis according to their allocated group at randomisation. Statistical tests will use a p-value less than 0.05 for significance. All statistical analysis procedures will be done using STATA 14 and R version 3.6.3. Baseline summary statistics (mean, standard deviation, percentage) will be calculated based on groups. Chisquare tests, T-tests will be performed to test the significance of the study variables. The odds ratio of the outcome variables for the post-assessment will be calculated using logistic regression modelling and $95 \%$ confidence intervals. We will analyse descriptive summaries of socio-demographic aspects and the scores of WHO-5, PHQ- 9 and MSPSS as the baseline and after one month.

\section{Discussion}

To the best of our knowledge, there have been no previous published study or trial that employs telephonic befriending for managing the psychological and social determinants of vulnerable groups, with a country-level focus. Before the main trial, we have planned a pilot trial that will provide preliminary evidence regarding the feasibility, acceptability, and efficacy of a telephonic structured befriending intervention. It will provide an understanding as to what extent a befriending intervention aids to reduce worry, unprecedented pressure and promote healthy coping to the challenges faced during the time of the pandemic. The analysis from the main trial will aim to establish whether there are benefits from a telephone befriending intervention compared with the control group during a pandemic. During the 
COVID-19 pandemic, although maintaining physical distance is a necessity, being socially connected and closely in dialogue has become the need for the day.

\section{Trial status}

This intervention trial is registered under Clinical Trial Registry India (ICMR-NIMS) on $27^{\text {th }}$ July 2020; with the registration number (CTRICTRI/2020/07/026834) and reference number (REF/2020/07/035397). The start date is $7^{\text {th }}$ August 2020 and the whole process is likely to be completed by $31^{\text {st }}$ July 2021 .

Recruitment will start from $1^{\text {st }}$ September 2020 and will be completed by $10^{\text {th }}$ September 2020 .

\section{Abbreviations}

COVID-19: coronavirus disease 2019

WHO-5: World Health Organization-Five Well-being Index

PHQ-9: Patient Health Questionnaire

MSPSS-12: Multidimensional Scale of Perceived Social Support

DDUGKY: Deen Dayal Upadhyaya Grameen Kaushalya Yojana

NIRDPR: National Institute for Rural Development and Panchayath Raj

PIA: Project Implementing Agency Declarations

\section{Declarations}

\section{Ethics approval and consent to participate}

Ethics committee approval was obtained from the Rajagiri Institutional Review Board (IRB) of Rajagiri College of Social Sciences (Autonomous) with reference number: RIRB/2004. For the baseline assessment, if participants start the questionnaire, it will be considered as the first level of consent. Oral consent will be taken before allocating the participants to the intervention or control arm. This oral consent will be audio recorded with their permission. Participants are free to discontinue from the intervention anytime

\section{Consent for publication}

Consent of publication is not applicable because in this paper we do not publish any individual data.

\section{Availability of data and materials}

Not applicable 


\section{Competing interests}

The authors declare that they have no competing interests

\section{Funding}

The technical assistance for the study is funded by Rajagiri College of Social Sciences (Autonomous); Information and dissemination of the project including publication will be funded by UK-India Education and Research Initiative funded by UGC and British Council Project (reference number - UGC-UKIERI- 201617-089, F. NO. 184-3/2017 (IC)). Funders have no role in study design, data collection and analysis, decision to publish, or preparation of the manuscript.

\section{Author's contributions}

SMD conceptualized, designed and drafted the manuscript of the protocol. KP revised the manuscript and made valuable contributions to the manuscript and its conclusions. AMB, LS, NC and SIP has contributed to the discussion and content of the paper. All authors read and approved the final manuscript.

\section{Acknowledgements}

The authors wish to thank all the students of the DDUGKY programme of Rajagiri College of Social Sciences who were part of the data collection. Authors also express their gratitude to all the DDUGKY staff who were part of the project in collecting the data and delivering the telephonic intervention. Authors would like to specifically mention Mr Rajeev SR, Coordinator, DDUGKY centre of Rajagiri College for his special effort in coordinating and managing the project within the centre.

\section{References}

1. Wikipedia. Covid-19 pandemic in India. Retrieved on 1st August 2020 from https://en.wikipedia.org/wiki/COVID-19_pandemic_in_India

2. World Health Organization. Archived: WHO Timeline-COVID-19. 27th April 2020. Retrieved on 1st August 2020 from https://www.who.int/news-room/detail/27-04-2020-who-timeline--covid-19

3. Wikipedia. Covid-19 pandemic in Kerala. Retrieved on 1st August 2020 from https://en.wikipedia.org/wiki/COVID-19_pandemic_in_Kerala

4. Zandifar A, Badrfam R. Iranian mental health during the COVID-19 epidemic [published online ahead of print, 2020 Mar 4]. Asian J Psychiatr. 2020;51:101990. doi:10.1016/j.ajp.2020.101990

5. Wang C, Pan R, Wan X, et al. Immediate Psychological Responses and Associated Factors during the Initial Stage of the 2019 Coronavirus Disease (COVID-19) Epidemic among the General Population in China. Int J Environ Res Public Health. 2020;17(5):1729. Published 2020 Mar 6.

doi:10.3390/ijerph17051729 
6. Brooks SK, Webster RK, Smith LE, Woodland L, Wessely S, Greenberg N. et al. The psychological impact of quarantine and how to reduce it: rapid review of the evidence. Lancet. 2020; 395: 912-920

7. Kochhar AS, Bhasin R, Kochhar GK, Dadlani H, Mehta VV, Kaur R, Bhasin CK. Lockdown of 1.3 billion people in India during Covid-19 pandemic: A survey of its impact on mental health. Asian J Psychiatr. 2020 Jun;54 102213. doi:10.1016/j.ajp.2020.102213. PMID: 32599544; PMCID: PMC7301781.

8. Economic times-Indiatimes. Job loss most severe immediate impact of COVID-19: survey. Published on11th June 2020. Retrieved on 19th July 2020 from https://economictimes.indiatimes.com/jobs/job-loss-most-severe-immediate-impact-of-covid-19survey/articleshow/76323649.cms

9. Dar KA, Iqbal N, Mushtaq A. Intolerance of uncertainty, depression, and anxiety: Examining the indirect and moderating effects of worry. Asian J Psychiatr. 2017; 29, 129-133. https://doi.org/10.1016/j.ajp.2017.04.017

10. Ministry of Rural development. Deen Dayal Upadhyaya Grameen Kaushalya Yojana. Retrieved on 20th July 2020 from http://ddugky.gov.in

11. Patel V, Kleinman A. Poverty and common mental disorders in developing countries. Bull World Health Organ. 2003;81(8):609-615.

12. McManus S, Meltzer H, Brugha T, Bebbington P, Jenkins R. Leeds: The NHS Information Centre for health and social care. Adult Psych Morbid in England. 2007; DOI: 10.13140/2.1.1563.5205

13. Lehtinen V, SohIman B, Kovess-Masfety V. Level of positive mental health in the European Union: results from the Eurobarometer 2002 survey. Clin Pract Epidemiol Ment Health. 2005;1:9. Published 2005 Jul 21. doi:10.1186/1745-0179-1-9

14. Sagar R, Dandona R, Gururaj G, Dhaliwal R S, Singh A, Ferrari A, et.al. The burden of mental disorders across the states of India: the Global Burden of Disease Study 1990-2017. The lancet Psych. 2020; 7(2):148-161. https://psycnet.apa.org/doi/10.1016/S2215-0366(19)30475-4

15. Mead N, Lester H, Chew-Graham C, Gask L, Bower P. Effects of befriending on depressive symptoms and distress: systematic review and meta-analysis. Br J Psychiatry. 2010;196(2):96-101. doi:10.1192/bjp.bp.109.064089

16. Horton TV, Wallander JL. Hope and social support as resilience factors against psychological distress of mothers who care for children with chronic physical conditions. Rehabil Psychol. 2001; 46(4):382-399.

17. Van Bortel T, Basnayake A, Wurie F, et al. Psychosocial effects of an Ebola outbreak at individual, community and international levels. Bull World Health Organ. 2016;94(3):210-214. doi:10.2471/BLT.15.158543

18. Casale M, Wild L, Cluver L, Kuo C. Social support as a protective factor for depression among women caring for children in HIV-endemic South Africa. J Behav Med. 2015;38(1):17-27. doi:10.1007/s10865-014-9556-7

19. Harandi TF, Taghinasab MM, Nayeri TD. The correlation of social support with mental health: A metaanalysis. Electron Physician. 2017;9(9):5212-5222. Published 2017 Sep 25. doi:10.19082/5212 
20. Buttorff C, Hock R S, Weiss HA, Naik S, Araya R, Kirkwood BR, Chisholm D, Patel V. Economic evaluation of a task-shifting intervention for common mental disorders in India. Bull World Health Organ; (2012). 90(11), 813-821.

21. Topp CW, Østergaard SD, Søndergaard S, Bech P. The WHO-5 Well-Being Index: a systematic review of the literature. Psychother Psychosom. 2015;84(3):167-176. doi:10.1159/000376585.

22. WHO. 1998. Wellbeing measures in primary health care/the Depcare project. WHO regional office for Europe: Copenhagen

23. Kroenke K, Spitzer RL, Williams JBW. The PHQ-9. J Gen Intern Med. 2001;16(9), 606-613. doi:10.1046/j.1525-1497.2001.016009606.x

24. Zimet GD, Dahlem NW, Zimet SG, Farley GK. The Multidimensional Scale of Perceived Social Support. J Pers Assess. (1988); 52(1), 30-41.

\section{Figures}




\begin{tabular}{|c|c|c|c|c|c|c|c|}
\hline \multirow[b]{3}{*}{ TIMEPOINT $^{* *}$} & \multicolumn{7}{|c|}{ STUDY PERIOD } \\
\hline & \multirow{2}{*}{$\begin{array}{c}\text { Enrolment } \\
\text { September } \\
2020 \\
\end{array}$} & \multirow{2}{*}{$\begin{array}{c}\text { Allocation } \\
\text { September } \\
2020 \\
\end{array}$} & \multicolumn{4}{|c|}{ Post-allocation } & \multirow{2}{*}{$\begin{array}{c}\text { Close-out } \\
\text { November } \\
2020 \\
\end{array}$} \\
\hline & & & $W K^{* 1}$ & $W K^{*} 2$ & $W K^{*} 3$ & $W K^{*} 4$ & \\
\hline \multicolumn{8}{|l|}{ ENROLMENT: } \\
\hline Eligibility screen & $\mathrm{X}$ & & & & & & \\
\hline Informed consent & $X$ & & & & & & \\
\hline Allocation & & $x$ & & & & & \\
\hline \multicolumn{8}{|l|}{$\begin{array}{r}\text { Experimental } \\
\text { Group }\end{array}$} \\
\hline \multicolumn{8}{|l|}{$\begin{array}{r}R E \text { ECH (Resiliency } \\
\text { Engagement and Care } \\
\text { in Health) }\end{array}$} \\
\hline \multicolumn{8}{|l|}{ Control Group } \\
\hline \multicolumn{8}{|l|}{$\begin{array}{l}\text { General Enquiry } \\
\text { Telephone calls }\end{array}$} \\
\hline \multicolumn{8}{|l|}{ ASSESSMENTS: } \\
\hline \multirow{3}{*}{$\begin{array}{l}\text { Baseline: } \\
\text { Sociodemographic data, } \\
\text { economic and } \\
\text { occupational status } \\
\text { Outcome: } \\
\text { Mental Wellbeing (WHO } \\
\text { wellbeing index), } \\
\text { Depression (Patient } \\
\text { health questionnaire), } \\
\text { Perceived social Support } \\
\text { (MSPS-12) }\end{array}$} & $\mathrm{X}$ & $X$ & & & & & \\
\hline & & & $X$ & & & $X$ & $X$ \\
\hline & & & $X$ & & & $\mathrm{X}$ & $X$ \\
\hline
\end{tabular}

Figure 1

Assessment Schedule of Enrollment, Interventions and assessment. Fig Schedule of enrollment, interventions, and assessment (formatted on the basis of the Standard Protocol Items Recommendations for Interventional Trials [SPIRIT] 2013 template) *WK = Week 


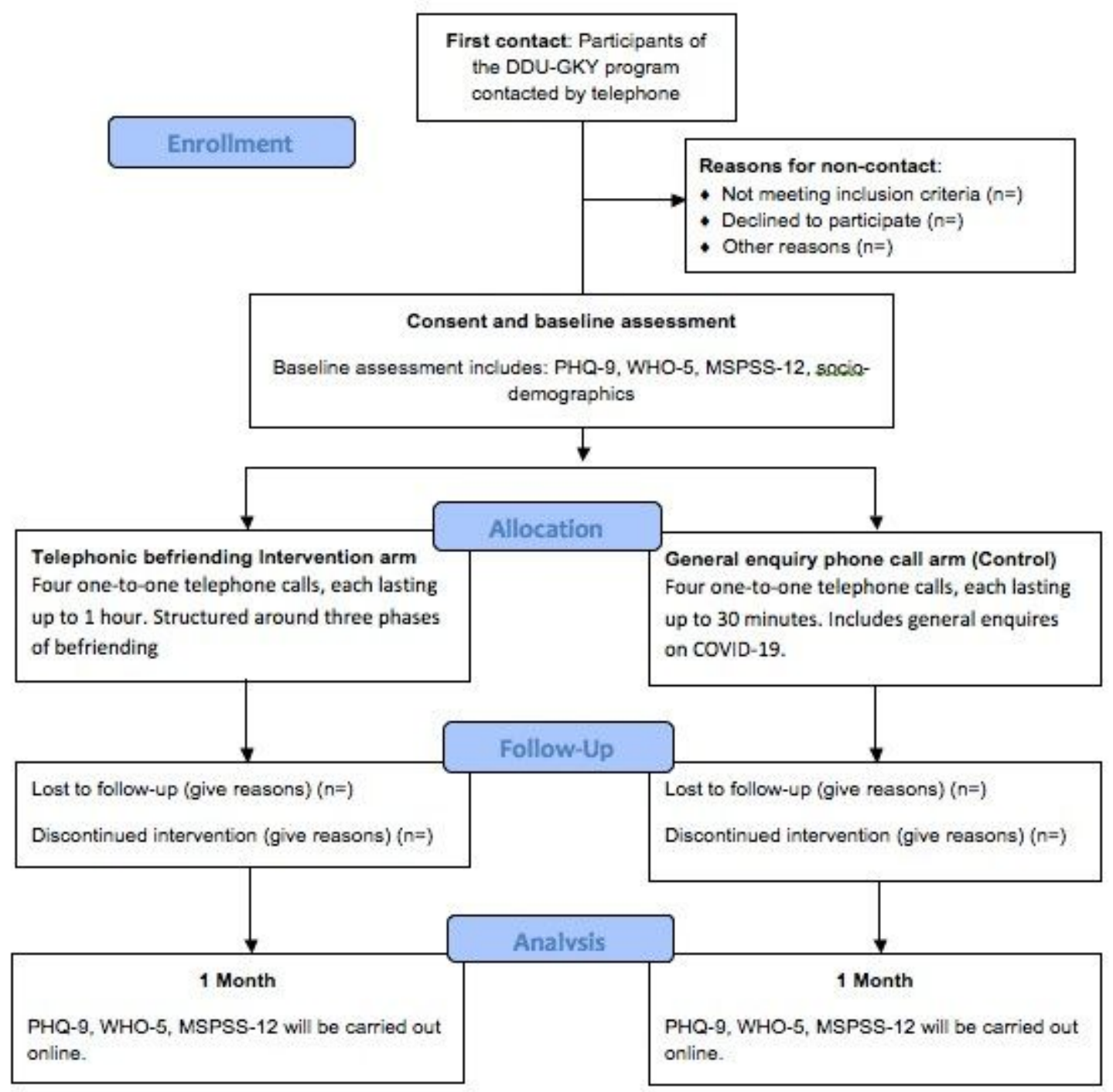

Figure 2

Process of the trial

\section{Supplementary Files}

This is a list of supplementary files associated with this preprint. Click to download.

- spiritchecklist.doc 